\title{
Carrying capacity - a capricious construct
}

\author{
Roger Cropp \\ Griffith School of Environment, Griffith University, Nathan, Queensland, 4111, Australia \\ J. Norbury \\ Mathematical Institute, University of Oxford, Andrew Wiles Building, ROQ, Woodstock \\ Road, Oxford OX2 6GG, UK
}

\begin{abstract}
The concept of a carrying capacity of an environment for a population has been an effective way of introducing a connection between populations and their environment. This approach has been particularly effective in heuristic Lotka-Volterra models of competition and predation, that provide a basis for theoretical considerations of population interactions. However, the concept has proved less useful for interactions such as mutualism.

The textbook Lotka-Volterra model of mutualism represents the interaction between two populations at the same trophic level that are each limited by the (negative) carrying capacities of the environment. The model predicts that obligate mutualist populations will either go extinct or become infinite. The classical fail diagram of obligate mutualist population interactions can be remedied by explicitly accounting for resources.

The inclusion of explicit connections to the environment through resource representation and accounting for each population shows that the equivalent of the classic fail diagram is actually a sensible solution. A resource-based modelling framework saves the Lotka-Volterra model, allowing the classic population interactions of competition, predation and mutualism to be considered in a single unified framework.
\end{abstract}

Email address: r.cropp@griffith.edu.au (Roger Cropp)

Preprint submitted to Ecological Modelling

March 15, 2019 
Keywords: carrying capacity, obligate mutualism, explicit resource accounting, consumer-resource model, Lotka-Volterra model

\section{Introduction}

The concept of the carrying capacity of an environment for a population is mentioned in most text books on ecology, and has played a central role in our conceptualisation of how populations interact, often articulated through simple

5 Lotka-Volterra population models. Carrying capacity Lotka-Volterra models of competition and predation interactions between two populations grace the pages of most ecology textbooks, and provide a useful introduction to the key concepts of how populations interact with each other.

However, the definition of carrying capacity is far from consistent or clear [1]. In particular, the Lotka-Volterra model of obligate mutualism assumes negative carrying capacities, an assumption that appears to preclude any meaningful interpretation of the relationship between the populations and their environment. This model is well-known for producing the unhelpful solution that obligate mutualist populations cannot coexist [2, p49-70] [3, p99]. This theoretical result

15 is in stark contrast to empirical results that suggest that over $80 \%$ of species on Earth are involved in mutualism relationships, many of them obligate [4, 5]. Interestingly, this inconsistency between empirical and theoretical research appears not to be generally regarded as a paradox in the same way that a similar inconsistency became the Paradox of the Plankton [6].

The classic Lotka-Volterra representation of obligate mutualism is often represented by a phase plane diagram such as Figure 1(a) (but other representations are possible [7). This diagram indicates that only two outcomes of obligate mutualism are possible - both populations go extinct, or both populations become infinity large.

Cropp and Norbury 8 show that carrying capacity is equivalent to conservation of mass for a two-trophic level Lotka-Volterra model of one predator and one prey (i.e. plant-herbivore), but that this is not the case for a three-trophic 


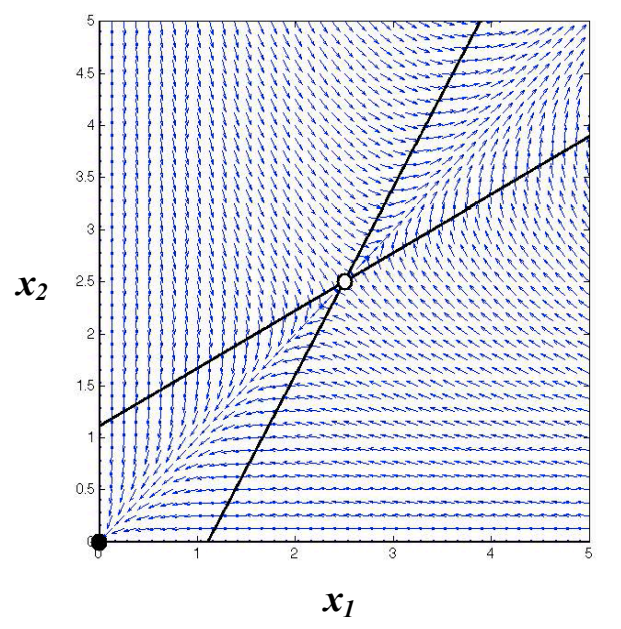

Figure 1: The traditional diagram of obligate mutualist population interactions. The solid lines are zero isoclines, where a population does not grow, the open circles show unstable equilibrium points, and filled circles show stable equilibria. The blue arrows indicate how populations will change, so populations starting from any given sizes will follow the arrows. The classic fail diagram of obligate mutualism for Lotka-Volterra models is shown, in which each axis shows the size of a heterotrophic obligate mutualist population. Here, either both populations go extinct, or both grow unboundedly.

level predation model (i.e. plant-herbivore-carnivore). The use of carrying capacity in a three-trophic level model could allow the population at the highest trophic level to be unbounded. This suggests that carrying capacity may be a useful mechanism for linking a population to the finite resources of its environment only for specific population interactions, as in some cases it allows infinite resources.

We show in this paper that carrying capacity also fails to validly link obligate mutualist populations to the constraints of their environment, resulting in "an orgy of mutual benefaction" [2, in an environment that also has effectively infinite resources. This is succinctly captured in the traditional phase plane diagram (Figure 1) that we refer to as the classic fail diagram of the Lotka-Volterra theory of population interactions involving obligate mutualism [9, 10, 11]. We 40 show that when obligate mutualist populations interact in an environment with 
finite resources, this diagram is subtly changed so that it represents a valid, sensible solution in which two obligate mutualist populations stably coexist.

An obligate mutualist population requires the presence of another, facilitating, population in addition to the resource(s) it consumes in order to grow. Obligate mutualisms between heterotrophs are commonly found in nature, for example protection obligations such as the sea anemone and the clown fish or the goby and shrimp [12] (see [13] for descriptions of 20 or so other such mutualist interactions, and 14 for over 30 descriptions of uni- and bi-directional mutualisms viewed from a consumer-resource perspective).

The key to the approach that we take in this manuscript is the fundamental principle that, rather than abstract the resources available to each population into a carrying capacity we require that every population has its resources explicitly represented and accounted for in the model. This is the basic resource-based framework for modelling ecosystems set out in Cropp and Norbury [15] that has proved useful in synthesising population interactions from competition through mixotrophy to facultative mutualism and predation [16].

\section{Resource-based Models}

The approach we take is based on two key tenets: each population has the resource it requires to grow explicitly represented in the model; and a common conserved currency is used to represent all populations and resources. This ensures that costs and benefits to each population are directly comparable. Typically in ecosystem models the key limiting resource is used as the common conserved currency. Although few ecosystems exactly conserve this resource (with notable exceptions such as the current lingua franca of marine ecosystem research, the sealed mesocosm [17]) most ecosystems conserve their limiting resource very tightly, typically recycling over 90\% [18]. Implementing exact conservation of limiting nutrient (but allowing all other nutrients and energy to flow freely through the system boundaries), and 100\% recycling, is a good first approximation to most ecosystems. This approach has been shown to 
work effectively for interactions between autotrophs (such as plant protections [19, 20, 21]) and between trophic levels (for example, pollination [5, 22, 23]) within a simple unified theory of population interactions in ecology [15].

(a)

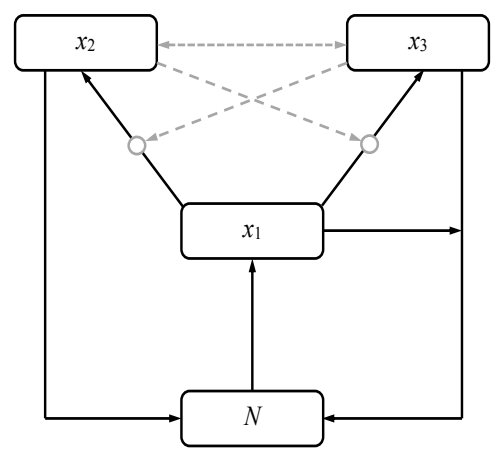

(b)

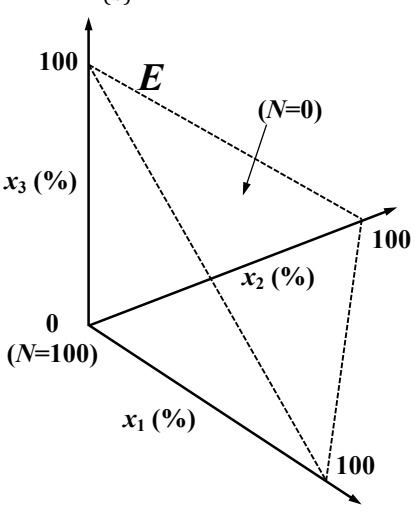

Figure 2: (a) Food web diagram showing the three living populations $x_{1}, x_{2}, x_{3}$, the inorganic nutrient pool $N$, and the flows of limiting nutrient. The solid arrows show direct flows of limiting resource due to photosynthesis, grazing, mortality and excretion. The dashed line ending in a circle connecting a population to a flow indicates the influence of a beneficial mutualist interaction by one population in facilitating a flow of resource for the other. The dotted line between $x_{2}$ and $x_{3}$ represents how these populations influence each other via competition without a direct exchange of mass. (b) The ecospace $(E)$ showing the percentages of the total limiting resource that could possibly be sequestered by the three living populations. Note that the percentage of limiting resource $N$ (as this is commonly a limiting nutrient) is also represented in this diagram due to the relationship $N=100-x_{1}-x_{2}-x_{3}$. Here, all populations are represented by their share of the cycling, limiting resource in the food web.

The model ecosystem is open to flows of energy and other nutrients, and we make no assumptions about stochiometry, nor eliminate the possibility that ecosystems may have multiple limiting nutrients that may limit the system at varying times. The total amount of limiting resource in the system in Figure 2(a) is taken to be constant, which allows us to define the ecospace $(E)$ in Figure 2(b); this figure shows the limiting resource $N$ implicitly as well as the populations explicitly. This finite phase space $E$ contains all ecologically-valid 
solutions to the model: our solutions to a food web defined on $E$ remain in $E$ for all time. Further, the resource space occupies the same space as $E$, where in this case the inorganic limiting nutrient $N$ is the resource for $x_{1}$, and $x_{1}$ is the resource for $x_{2}$ and $x_{3}$. Mutualism involves direct flows of resource, such as a population consuming its resource, and indirect flows of resource in which a population receives a benefit from its interaction with another population, but does not gain that benefit directly from the benefactor. Direct flows of mass are shown by solid lines in Figure 2(a) and indirect flows to the obligate mutualist populations in our example are shown by the dashed lines.

\section{The Obligate Mutualism Model}

We consider the classic scenario of obligate mutualism between two heterotroph populations $x_{2}$ and $x_{3}$, at the same trophic level, that feed on a common resource autotroph population $x_{1}$, that consumes an inorganic form of the limiting nutrient contained in the resource pool $N$. The limiting nutrient serves as the currency of the model. Here, as in all classical models of obligate mutualism, $x_{2}$ and $x_{3}$ depend upon the presence of each other to grow, and we note that allowing both populations to feed on the same resource is a simplification that does not compromise the heuristic value of the model:

$$
\begin{aligned}
\dot{x}_{1}= & x_{1} f_{1}\left(x_{1}, x_{2}, x_{3}, N\right)=\left(r_{1} N-m_{1}-a_{11} x_{1}-a_{12} x_{3} x_{2}-a_{13} x_{2} x_{3}\right) x_{1}, \\
\dot{x}_{2}= & x_{2} f_{2}\left(x_{1}, x_{2}, x_{3}\right)=\left(r_{2}+a_{21} x_{3} x_{1}-a_{22} x_{2}-a_{23} x_{3}\right) x_{2}, \\
\dot{x}_{3}= & x_{3} f_{3}\left(x_{1}, x_{2}, x_{3}\right)=\left(r_{3}+a_{31} x_{2} x_{1}-a_{32} x_{2}-a_{33} x_{3}\right) x_{3}, \\
\dot{N}= & -r_{1} N x_{1}+m_{1} x_{1}-r_{2} x_{2}-r_{3} x_{3}+a_{11} x_{1}^{2}+a_{22} x_{2}^{2}+a_{33} x_{3}^{2} \\
& +\left[\left(a_{12}-a_{21}\right)+\left(a_{13}-a_{31}\right)\right] x_{1} x_{2} x_{3}+\left(a_{23}+a_{32}\right) x_{2} x_{3} .
\end{aligned}
$$

Here $r_{1} N x_{1}$ represents the nutrient-limited growth of the autotroph $x_{1}$, and $m_{1} x_{1}, r_{2} x_{2}$ and $r_{3} x_{3}$ represent the density-independent mortality of $x_{1}$, $x_{2}$ and $x_{3}$ respectively. Similarly, $a_{11} x_{1}^{2}, a_{22} x_{2}^{2}$, and $a_{33} x_{3}^{2}$ represent the densitydependent mortality of $x_{1}, x_{2}$ and $x_{3}$ respectively. The obligate growth pro- 
cesses are grouped together in the $x_{1} x_{2} x_{3}$ terms, with the coefficients $a_{12}-a_{21}$ and $a_{13}-a_{31}$ in the final equation denoting the net outcomes, accounting for any losses in the process due to sloppy feeding, respiration, etc. Note that as these terms represent the feeding of $x_{2}$ and $x_{3}$ on $x_{1}$ (facilitated by $x_{3}$ and $x_{2}$ respectively) mass balance requires that $a_{12}>a_{21}$ and $a_{13}>a_{31}$ to ensure that the predator (grazer) does not gain more mass than the prey (plant) loses. The last term in the equation for $\dot{N},\left(a_{23}+a_{32}\right) x_{2} x_{3}$, represents the net outcome of competition/mutualism between $x_{2}$ and $x_{3}$.

This model represents a simple, generic environmental scenario, where the net exchange of resource (in this case a limiting nutrient) across the boundary of the ecosystem is zero (i.e. immigration + nutrient inflows $=$ emigration + nutrient outflows). The system has been scaled by the total limiting nutrient, so that the relation $N+x_{1}+x_{2}+x_{3}=1$ (i.e. $1 \equiv 100 \%$ of limiting nutrient) holds for all time for the limiting nutrient. Flows of other resources, such as sunlight, water, oxygen and other nutrients, are not restricted. This approach also provides an ideal model for mesocosm experiments, that are closed at least with respect to the limiting nutrient, and often to all nutrients, but are open to flows of energy.

The $r_{i}$ terms in Lotka-Volterra-like models such as (1) reflect how population $x_{i}$ interacts with the nutrient pool $N$, either as an autotroph for which growth on inorganic nutrient implies $r_{1}-m_{1}>0$, or as a heterotroph that only has mortality losses to the pool (in which case $r_{2}, r_{3}<0$ ). Similarly, the $a_{i j}$ terms measure the strength of interactions between populations, with the $a_{i i}$ terms representing density-dependent mortality, sometimes referred to as model closure terms as they include predation from unrepresented higher trophic levels. Generally, populations have a negative impact on each other, as resources sequestered in one population are not available to the other populations unless they have a predator-prey relationship, so the default is for the $a_{i j}$ coefficients to be preceded by minus signs.

The heterotroph populations $x_{2}$ and $x_{3}$ in (1) grow by consuming the autotroph population $x_{1}$. Here, the term $a_{21} x_{3} x_{1}$ reflects the obligation of the 
population $x_{2}$ on the presence of $x_{3}$ to be able to consume $x_{1}$ and grow, and vice versa for the obligate growth of $x_{3}$ on $x_{2}$. In accordance with convention in models of obligate mutualism, we incorporate into the model equations the fact that $x_{2}$ and $x_{3}$ consume $x_{1}$, and preface the $a_{21}$ and $a_{31}$ coefficients with a plus sign, allowing them to be defined as positive parameters in (1).

The dependence of one population on the presence of another to facilitate the consumption of resources and subsequent growth is observed in many mutualist interactions, for example between corals and fish [24], anemones and fish [25], corals and shrimp [26, and alpheid shrimp and gobies [27]. The growth terms that describe these interactions in models typically involve the product of the three populations that are required for growth - the resource (which may be inorganic nutrient), the obligating and the obligated populations. Thompson et al. 13] provide around twenty examples of such growth dependencies across eight mutualist interactions, mostly marine.

When a population, for example $x_{2}$, consumes a population $x_{1}$ as a resource and is obligated upon another population $x_{3}$ to be able to grow, its growth is a function of all three populations, that is $G\left(x_{2}\right) \propto g\left(x_{1}, x_{2}, x_{3}\right)$. We use the simplest possible form of this interaction for this analysis $\left(G\left(x_{2}\right)=\right.$ $\left.a_{21} x_{1} x_{2} x_{3}\right)$, but note that Thompson et al. [13] used a saturating form $\left(G\left(x_{2}\right)=\right.$ $\left.a_{21} x_{1} x_{2}\left[x_{3} /\left(x_{3}+\kappa_{3}\right)\right]\right)$ in their models. Here, $\kappa_{3}$ is a half-saturation constant of obligation that reflects the ability of the population $x_{2}$ to grow when its facilitating population level $x_{3}$ is low. When $x_{3}<\kappa_{3}, x_{2}$ 's growth rate increases roughly linearly with $x_{3}$; when $x_{3}>\kappa_{3}, x_{2}$ 's growth rate begins to saturate, and the presence of a larger facilitating population does not produce a commensurate increase in $x_{2}$ 's growth rate. In our formulation, both $x_{2}$ and $x_{3}$ are always obligate mutualists, each depending on the presence of the other to be able to grow, irrespective of the signs of $a_{23}$ and $a_{32}$. Mortality terms $r_{2} x_{2}$ and $-a_{22} x_{2}^{2}$ may be interpreted as linear and quadratic mortality which could include model closure effects (similarly $r_{3} x_{3}$ and $-a_{33} x_{3}^{2}$ ). The $a_{23} x_{2} x_{3}$ term is the net mutualist loss (or gain if the costs of providing an incentive for a mutualist benefit are less than the gain [14]) to $x_{2}$ arising from the presence of 
$x_{3}$ (and vice versa).

Note that the choice of functional form for the obligation interaction is not critical to the attributes of the model (1), in contrast to models such as [28]. Further, the Lotka-Volterra model is a good numerical approximation to the saturating term models used by Thompson et al. [13] when $\kappa_{3}>1$ and $\kappa_{2}>1$. While we have used Lotka-Volterra equations as the basis for this model, the results are not dependent upon the use of these equations.

The form of equation (1) ensures that populations that start with $x_{i}(0)>0$ keep $x_{i}(t)>0$ in $E$, and cannot become negative. For model validity we require $\sum_{i=1}^{n} x_{i}<1$; this is ensured by stipulating that $N(t) \geq 0$. This is implemented by choosing parameter ranges so that when the resource pool is empty flows 175 of limiting resource from the pool cannot exceed the flows into the pool from mortality (i.e. $\dot{N} \geq 0$ when $N=0$ ). Equivalently, this means that when the resource pool is empty the total of the living populations cannot increase $\left(\sum \dot{x}_{i} \leq 0\right.$ when $\left.\sum x_{i}=1\right)$.

The parameter set is also subject to ecological versimilitude conditions. These stem from the basic principle that populations are not immortal, that is, if they do not have any resources they cannot grow, and that we do not consider unviable populations, that is, populations that cannot grow even when the world is full of their resources. These conditions are explained in detail in [15], but briefly, they require that:

$$
\begin{array}{r}
\left.f_{1}\right|_{N=1}>0>\left.f_{1}\right|_{N=0}, \\
\left.f_{2}\right|_{x_{1}+x_{3}=1, x_{1} \neq 0 \neq x_{3}}>0>\left.f_{2}\right|_{x_{1}=0 \text { and } / \text { or } x_{3}=0}, \\
\left.f_{3}\right|_{x_{1}+x_{2}=1, x_{1} \neq 0 \neq x_{2}}>0>\left.f_{3}\right|_{x_{1}=0 \text { and } / o r} x_{2}=0,
\end{array}
$$

where $f_{1}, f_{2}$ and $f_{3}$ are defined in equation (1). These conditions produce the parameter constraints: 


$$
\begin{array}{r}
r_{1}-m_{1}>0, m_{1}+a_{11}>0, m_{1}+a_{12}>0, \text { and } m_{1}+a_{13}>0, \\
r_{2}<0, a_{21}>a_{23}, \text { and } a_{22}>0, \\
r_{3}<0, a_{31}>a_{32}, \text { and } a_{33}>0 .
\end{array}
$$

Additionally, local mass balance, such that a predator cannot gain more mass than its prey loses, requires that $a_{12}>a_{21}$ and $a_{13}>a_{31}$. Note that $a_{23}<0$ and $a_{32}<0$ indicate a beneficial mutualist interaction between $x_{2}$ and $x_{3}$. When negative, the magnitude of these parameter values in particular are constrained by the condition $\dot{N}>0$ when $N=0$ noted above. All parameter sets that comply with these criteria will produce sensible models of obligate mutualism for equation (1). The parameter values used in the simulations comply with these conditions, but many other parameter sets that comply with the conditions will produce similar results (see the caption to Figure 3 for the actual parameter values used).

\section{Equilibrium Points}

The model (1) has four equilibrium points: a point $\left(C_{0}\right)$ at the origin of the ecospace where all populations are extinct; an autotroph-only point $\left(C_{1}\right)$; and two equilibria where all three populations coexist, one stable $\left(C_{3 S}\right)$ and one unstable $\left(C_{3 U}\right)$. Figure 3 (b and c) shows that these two equilibrium points lead to Allee effects for the $x_{2}$ and $x_{3}$ populations. Recall that the defining attribute of obligate mutualism for the $x_{2}$ and $x_{3}$ populations is that the equilibrium points $\left\{x_{1}^{*}, x_{2}^{*}, 0\right\}$ and $\left\{x_{1}^{*}, 0, x_{3}^{*}\right\}$ lie outside the ecospace, since such obligate mutualist populations $x_{2}$ and $x_{3}$ cannot exist when their obligate partner is absent.

The stabilities of the equilibrium points are determined by the signs of the eigenvalues of the Jacobian matrix defined by the partial derivatives of the righthand side of (1) evaluated at the appropriate equilibrium point. The origin 
point $C_{0}=\{0,0,0\}$ has the eigenvalues:

$$
\lambda_{C_{0}(1)}=r_{1}-m_{1}>0, \quad \lambda_{C_{0}(2)}=r_{2}<0, \quad \text { and } \quad \lambda_{C_{0}(3)}=r_{3}<0,
$$

and is an unstable saddle point. The autotroph point $C_{1}$ is located at $\{0<$ $\left.x_{1}^{*}=\left(r_{1}-m_{1}\right) /\left(r_{1}+a_{11}\right)<1\right\}$ and has the eigenvalues:

$$
\lambda_{C_{1}(1)}=-\left(r_{1}-m_{1}\right)<0, \quad \lambda_{C_{1}(2)}=r_{2}<0, \quad \text { and } \quad \lambda_{C_{1}(3)}=r_{3}<0,
$$

and is a stable node. This point lies on the $x_{1}$ axis within $E$, and is stable if $x_{2}$ and $x_{3}$ are obligate heterotrophs. Here $\left(r_{1}-m_{1}\right) /\left(r_{1}+a_{11}\right)$ for $a_{11}>0$ might be interpreted as the carrying capacity of the environment for $x_{1}$ when the mutualist populations $x_{2}$ and $x_{3}$ are absent.

The algebraic expressions describing the locations of the two coexistence points $C_{3 S}$ and $C_{3 U}$ are complex and effectively uninformative, but may be simplified considerably if we consider the neutral theory case where the two heterotroph populations are functionally equivalent. This provides a neat analytical solution that we can use to check the numerical code for the symmetric case (shown in Figure 4(b)) as a limiting case of the asymmetric solution we show in Figure 3. The values of the stable $\left(x_{2 S}^{*}=x_{3 S}^{*}\right)$ and unstable $\left(x_{2 U}^{*}=x_{3 U}^{*}\right)$ equilibrium points are given by the two positive roots of:

$$
\begin{gathered}
\alpha x_{2}^{* 3}+\beta x_{2}^{* 2}+\gamma x_{2}^{*}+\delta=0, \quad \text { where: } \alpha=a_{21}\left(a_{12}+a_{13}\right), \quad \beta=2 r_{1} a_{21}, \\
\gamma=-\left(r_{1}-m_{1}\right) * a_{21}-\left(r_{1}+a_{11}\right) *\left(a_{22}+a_{23}\right), \text { and } \delta=r_{1} r_{2}+a_{11} r_{2} .
\end{gathered}
$$

${ }_{225}$ The values for $x_{1 U}^{*}$ and $x_{1 S}^{*}$ are given in terms of these positive roots $x_{2 U}^{*}$ and $x_{2 S}^{*}$

$$
x_{1 S}^{*}=\frac{\left(r_{2}-\left(a_{22}+a_{23}\right) x_{2 S}^{*}\right)}{a_{21} x_{2 S}^{*}}, \quad x_{1 U}^{*}=\frac{\left(r_{2}-\left(a_{22}+a_{23}\right) x_{2 U}^{*}\right)}{a_{21} x_{2 U}^{*}},
$$


where $x_{2 S}^{*}=x_{3 S}^{*}$ is the most positive root and $x_{2 U}^{*}=x_{3 U}^{*}$ is the least positive root of (6). Algebraic expressions for the eigenvalues of even this simplified point are uninformatively complicated, and are evaluated and checked numerically. Here $x_{1 S}^{*}$ is conceptually equivalent to the level of resource for $x_{2}$ and $x_{3}$ that defines the carrying capacity of the environment for these populations.

\section{Dynamical Properties of the Obligate Mutualist Model}

The typical properties of the model (1) may be understood by considering its zero isoclines, equilibrium points and vector fields. These are shown in Figure

2353 , for a parameter set (see figure caption for parameter values) that describes two obligate mutualist heterotrophs that each receive strong mutualist benefits from the other in addition to facilitation of their growth. The zero isosurfaces of the autotroph $x_{1}$ are shown in blue, and the zero isosurfaces of the obligate heterotrophs $x_{2}$ and $x_{3}$ are shown in yellow and green respectively. Figure 3(a) has the property that, as is a defining attribute of obligate populations, the heterotroph zero isosurfaces do not intersect the axes of the ecospace.

Figure 3(b) also shows the dynamical properties of the obligate heterotroph system (1) starting from two different initial conditions: the green trajectory starts with all populations small; and the red trajectory starts with the $x_{1}$ and $x_{3}$ populations small, and the $x_{2}$ population large. These very different starting conditions, in conjunction with the vector fields shown on the faces of $E$, support our numerical eigenvalue analysis that suggests the $C_{3 S}$ equilibrium point that we have designated as stable is indeed stable.

The vector fields of the vertical sections through $E$ in Figure 3(c), show the equilibrium points and the separatrix associated with the unstable point that gives the Allee effect [29]. Here, the vertical section $\left\{x_{1}, x_{2}=x_{3}\right\}$ passes through both the stable and unstable points, $C_{3 S}$ and $C_{3 U}$, showing a section of the autotroph zero isosurfaces (the diagonal lines) and a section of a heterotroph zero isosurface (the curved line). This and the intersection of the heterotroph zero isosurfaces further from the origin in Figure 3(d) reveal the Allee effects 
[29]. In each case, separating surfaces must exist between the regions of the ecospace where the initial conditions lead to the stable coexistence equilibrium points $C_{3 S}$ and the regions of the ecospace where the initial conditions lead to the stable equilibrium point $C_{1}$ where $x_{2}$ and $x_{3}$ are extinct. The separatrices, that separate populations of $x_{2}$ and $x_{3}$ that can survive from those that can't, must pass through $C_{3 U}$.

Figure 3(d) reveals the key advantage of an explicit and exact resource accounting framework. This horizontal section of the vector field, drawn at $x_{1}=x_{1 S}^{*}$, is equivalent to the classic fail diagram of Lotka-Volterra models of obligate mutualism (Figure 1(a)). We emphasise this similarity in Figure 4 where in panel (a) the $\left\{x_{1}, x_{2}\right\}$ phase space of the classic Lotka-Volterra model of obligate mutualism sits beside the $\left\{x_{2}, x_{3}\right\}$ section through the ecospace of the model (1) in panel (b). The arrangements of the zero isoclines and the vector fields of the two models are similar in Figure 4 , but the absence of an equilibrium point at the origin and the stability of the coexistence equilibrium point indicate the fundamentally different dynamical properties of the two solutions. This is a stark revelation of the profound effect of including resources in even the simplest heuristic models.

The Lotka-Volterra model assumes a constant (negative) carrying capacity 275 that must in some respect reflect the resources provided by the environment to sustain the populations, whereas our diagram represents a slice through the three-dimensional vector field at a constant resource level. The vector field pointing away from the origin between the two zero isoclines in Figure 4(b) appears to indicate that our model violates the requirement that $\dot{N}=-\sum \dot{x}_{i}>$ 0 when $N=0$. When the three-dimensional vector sum is evaluated, the vectors actually point into $E$ because of the sloping lid, confirming the critical role of the additional resource dimension, and that the model is ecologically sound. The reasons for this are explained in detail in [30. 


\section{Discussion}

285 resource representation and exact resource accounting, that explains the deficiencies of carrying capacity using the example of obligate mutualism. This population interaction has confounded attempts to explain it using Lotka-Volterra models using carrying capacity, but is neatly explained by a resource-based Lotka-Volterra model.

The simplest version of this framework is a Lotka-Volterra model that is closed to the cycling limiting nutrient in the food web (but open to energy and all other nutrients). We examine obligate mutualist population interactions, as these reproduce the classic Lotka-Volterra fail diagram. This is often cited to demonstrate that obligate mutualism cannot be sensibly examined in simple, heuristic models. This is perceived to be the Achilles heel of the Lotka-Volterra models that have proved so useful for gaining insights into other population interactions, in particular predation and competition [15].

The major result in this paper is that the classic fail diagram of the LotkaVolterra model of obligate mutualism may be saved if resources are explicitly represented rather than a carrying capacity assumed. This result is synthesised in Figure 4. A vector field (Figure 4(a)) produced by a two population model that ignores resources, that convinced theoreticians that Lotka-Volterra models of obligate mutualists produced unrealistic solutions, is in fact part of a valid solution when viewed as a constant-resource two-dimensional slice through the

three-dimensional state space in a system that does represent resources (Figure $4(\mathrm{~b}))$.

Our results show that it essentially the abstraction of a resource as a carrying capacity, that constrains a three-dimensional problem to two dimensions, that is the cause of the problem. In previous work, we showed that particular mutualist processes such as plant-pollinator interactions and mutualist autotrophs can be successfully modelled in this framework [30.

Explicit and exact limiting resource accounting can be implemented in Lotka- 
Volterra (and most ecosystem) models without substantially increasing their

\section{Conclusions}

This paper demonstrates that explicit resource accounting for a finite total amount of limiting resource cycling on a closed food web saves the classic Lotka-Volterra model of obligate mutualism. We examine two interacting obligate heterotroph mutualists specifically because that model produces isoclines

tude. The explicit modelling of a limiting resource and a resource pool does, however, lead to more complicated higher-trophic level models. Here, heterotroph interactions require the modelling of their prey populations, obviating the mathematical analysis advantages of two-population systems, but this is outweighed by the explanatory ability of the model.

The explicit resource framework [15] allows obligate mutualism to be considered alongside the usual Lotka-Volterra models of competition and predation. We have used Lotka-Volterra-like obligation terms in our example model as these are the simplest form, but note that our results are not dependent on the use of such terms - qualitatively similar results are obtained with Holling Type II (saturating) forms which can also smoothly transition from facultative to obligate mutualism [22. Similarly, the resource-based approach provides sensible solutions for many different forms of mutualist interactions, with different interactions represented by very different functional forms. Cropp and Norbury [15, 16, 23, 20, 22, 30] present examples of resource-based Lotka-Volterra and other models that successfully explain: facultative mutualism; obligate mutualism interactions between autotrophs, plants and pollinators, and heterotrophs; and transitions from facultative to obligate mutualism, and from non-Allee to Allee solutions. and a vector field identical to the classical fail diagram of the obligate mutualism Lotka-Volterra example. However, the interior equilibrium point has the opposite stability. The resource-based model produces a different (and valid) so- 
lution while retaining all the other properties of the classic Lotka-Volterra model of obligate mutualism that use carrying capacity. This demonstrates that it is the inclusion of explicit resource accounting that changes the "silly" [3] solution of the carrying capacity model into the valid solution of the resource-based equivalent.

Loreau [31] suggests that Lotka-Volterra models are unable to produce useful answers for obligate mutualism because they ignore fundamental mass-balance constraints. Similarly, Johnson 32 notes that laws of thermodynamics and conservation of matter structure ecosystems, and suggests that "An ecosystem perspective helps provide context for studies of mutualism. Studies that focus solely on two interacting species are unlikely to accurately capture the dynamics of species interactions in nature because they miss critical feedbacks that emerge 355 from constraints imposed by the abiotic environment". We support these statements here with a simple model that includes mass conservation for the limiting nutrient that cycles through an abiotic pool. Our contention is that the failure of the Lotka-Volterra model of obligate mutualism is due to its use of carrying capacity.

Explicit, exact resource accounting provides a general solution to the familiar deficiencies of carrying capacity models. A resource-based approach allows not only the classic population interactions of competition, predation and both facultative and obligate mutualism, but also allows omnivory, mixotrophy and higher trophic level predation to be considered in a single unified framework [8, 15, 22]. This demonstrates the usefulness of including explicit finite resources for each population, and accounting for those resources exactly, and emphasises the importance of having a framework within which to construct the models.

\section{Acknowledgements}

The authors thank Brian Fath and Fabio Boschetti for their constructive comments on an earlier version of this manuscript. RC thanks Lincoln College, Oxford for providing funding to partly support this research. 


\section{References}

[1] J. Pastor, Mathematical Ecology of Populations and Ecosystems, WileyBlackwell, Chichester, 2008.

375 [2] R. May, Theoretical Ecology: Principles and Applications, Blackwell Science Inc, Oxford, 1981.

[3] J. Murray, Mathematical Biology, Springer-Verlag, Berlin, 2001.

[4] D. Boucher, The Biology of Mutualism, Croom Helm, London, 1985.

[5] J. Bronstein, Mutualism, Oxford University Press, New York, 2015.

[6] G. Hutchinson, The paradox of the plankton, The American Naturalist 95 (1961) 137-145.

[7] B. Fath, Network mutualism: Positive community level relations in ecosystems, Ecological Modelling 208 (2007) 56-67.

[8] R. Cropp, J. Norbury, Constructing ecologies, Journal of Theoretical Biology 294 (2012) 1-8.

[9] R. May, Stability and Complexity in Model Ecosystems, Princeton University Press, Princeton, New Jersey, 1973.

[10] S. Levin, The Princeton Guide to Ecology, Princeton University Press, Oxford, 2012.

[11] J. Holland, Population ecology of mutualism, in: J. Bronstein (Ed.), Mutualism, Oxford University Press, New York, 2015, pp. 133-158.

[12] P. Lyons, The benefit of obligate versus facultative strategies in a shrimpgoby mutualism, Behavioral Ecology and Sociobiology 67 (2013) 737-745.

[13] A. Thompson, R. Nisbet, R. Schmitt, Dynamics of mutualist populations that are demographically open, Journal of Animal Ecology 75 (2006) 12391251. 
[14] J. Holland, D. DeAngelis, A consumer-resource approach to the densitydependent population dynamics of mutualism, Ecology 91 (2010) 12861295.

[15] R. Cropp, J. Norbury, Population interactions in ecology: A rule-based approach to modeling ecosystems in a mass-conserving framework, SIAM Rev 57 (3) (2015) 437-465.

[16] R. Cropp, J. Norbury, Mixotrophy: the missing link in consumer-resourcebased ecologies, Theoretical Ecology 8 (2015) 245-260.

[17] U. Riebsell, J. Czerny, K. von Brockel, T. Boxhammer, J. Budenbender, M. Deckelnick, M. Fischer, D. Hoffmann, S. Krug, U. Lentz, A. Ludwig, R. Muche, K. Schulz, A mobile sea-going mesocosm system - new opportunities for ocean change research, Biogeosciences 10 (2013) 1835-1847.

[18] P. Vitousek, P. Matson, Nutrient cycling and biogeochemistry, in: S. Levin (Ed.), The Princeton Guide to Ecology, Vol. 1, Princeton University Press, Princeton New jersey, 2012, pp. 330-339.

[19] J. Bruno, Facilitation of cobble beach plant communities through habitat modification by spartina alterniflora, Ecology 5 (81) (2000) 1179-1192.

[20] R. Cropp, J. Norbury, Goby-shrimp mutualism: Costs and benefits of obligate versus facultative strategies, Ecological Complexity 36 (2018) 22-29.

[21] K. Gross, Positive interactions among competitors can produce species-rich communities, Ecology Letters 11 (2008) 929-936.

[22] R. Cropp, J. Norbury, Linking obligate mutualism models in an extended consumer-resource framework, Ecological Modelling 374 (2018) 1-13.

${ }_{420}$ [23] R. Cropp, J. Norbury, Obligate mutualism in an extended consumerresource framework, Yale Journal of Biology and Medicine 91 (2018) 375389. 
[24] J. Meyer, E. Schulz, G. Helfman, Fish schools - an asset to corals, Science 220 (1983) 1047-1049.

[25] R. Schmitt, S. Holbrook, Mutualism can mediate competition and promote coexistence, Ecology Letters 6 (2003) 898-902.

[26] J. Stachowicz, M. Hay, Mutualism and coral persistence: the role of herbivore resistance to algal chemical defense, Ecology 80 (1999) 2085-2101.

[27] I. Karplus, The association between gobiid fishes and burrowing alpheid shrimps, Oceanography and Marine Biology 25 (1987) 507-562.

[28] A. Bazykin, Nonlinear dynamics of interacting populations, World Scientific, Singapore, 1998.

[29] W. Allee, E. Bowen, Studies in animal aggregations: mass protection against colloidal silver among goldfishes, The Journal of Experimental Biology 61 (1932) 185-207.

[30] R. Cropp, J. Norbury, Obligate mutualism in a resource-based framework, SIAM Review.

[31] M. Loreau, From Populations to Ecosystems, Vol. 46 of Monographs in Population Biology, Princeton University Press, Woodstock, Oxfordshire, 2010.

[32] N. C. Johnson, Mutualisms and ecosystem-level processes, in: J. Bronstein (Ed.), Mutualism, Oxford University Press, New York, 2015, pp. 221-238. 

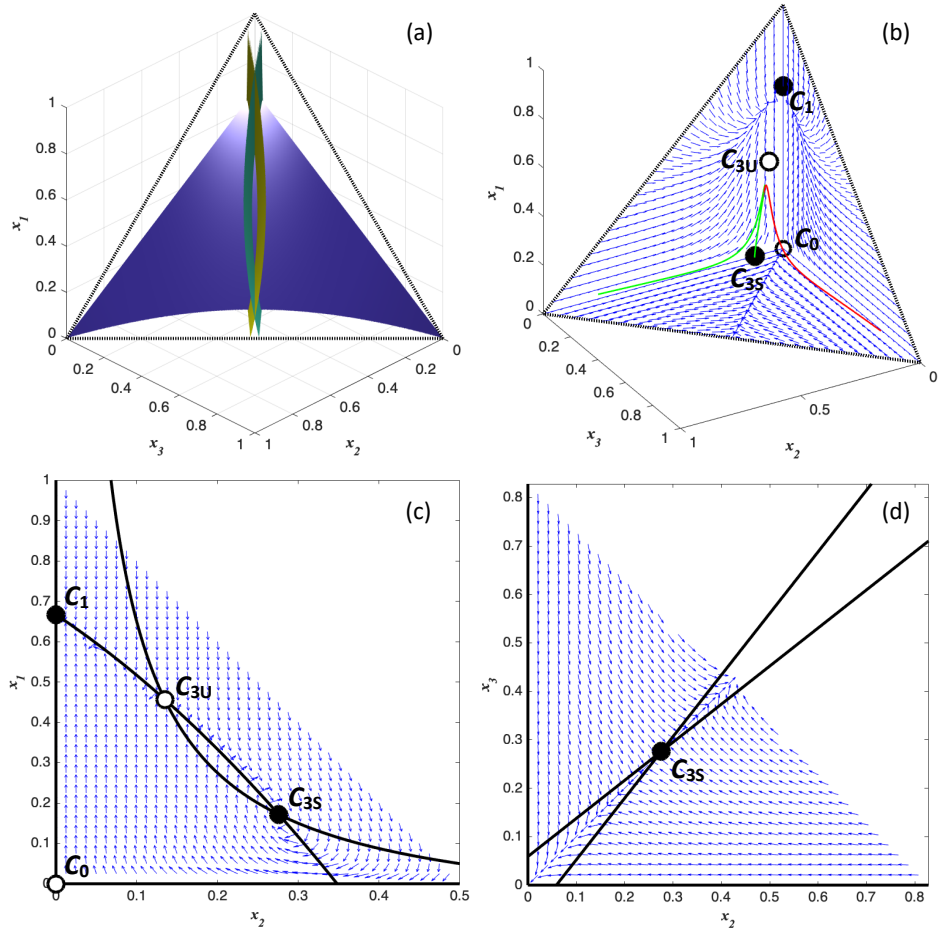

Figure 3: Ecospace diagram for a symmetric case of obligate mutualism between heterotrophs $x_{2}$ and $x_{3}$ where each is obligated to the other in order to feed on the autotroph $x_{1}$, but have asymmetric mutualist interaction coefficients $a_{23}$ and $a_{32}$. Each of $x_{2}$ and $x_{3}$ receives a strong net mutualist benefit from the other population, for instance as a mutual protection. Panels are: (a) $f_{i}=0$ isosurfaces for $x_{1}$ (blue), $x_{2}$ (brown), and $x_{3}$ (green); (b) trajectories from different initial conditions with the unstable origin and interior equilibrium points (open circles), and stable boundary and interior equilibrium points (filled circles) and scaled vector fields on the faces of $E$; (c) the scaled vector field and zero isoclines for $x_{1}$ (concave line) and $x_{2}$ (convex line) on the vertical section through (b) on the plane that passes through the origin and the stable coexistence equilibrium point; and (d) the scaled vector field and zero isoclines for $x_{2}$ (line running upper left to lower right) and $x_{3}$ (line running lower left to upper right) on the horizontal section through the interior equilibrium point where $x_{1}=x_{1}^{*}$. The equilibrium points are labelled $C_{0}, C_{1}, C_{3 U}$, and $C_{3 S}$ as in the text. The parameter values used to draw this figure are $\vec{r}=[1.000,-0.075,-0.075]^{T}, m_{1}=0.001$, $A=[0.50,1.25,1.25 ; 1.00,1.00,-1.10 ; 1.00,-1.10,1.00]$. 
(a)

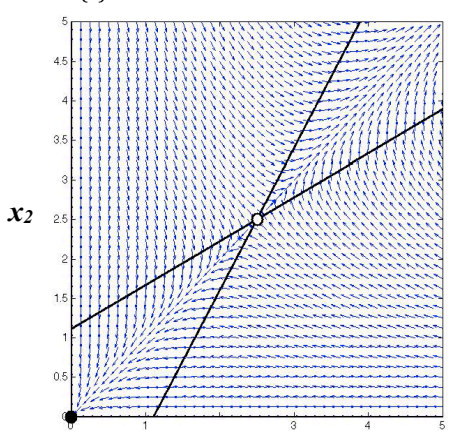

$x_{1}$ (b)

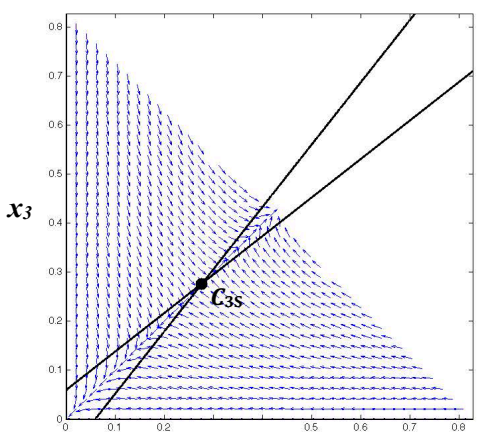

$x_{2}$

Figure 4: Ecospace diagrams with vector fields, zero isoclines, and equilibrium points for obligate mutualism between heterotrophs for : (a) the classic Lotka-Volterra model; and (b) the symmetric case for the resource-based Lotka-Volterra model. Details as for Figures 1(a) and 3(d) respectively. No stable positive coexistence equilibrium is possible in (a), but is in (b). Note equilibrium point $C_{3 S}$ is shown in (b) as in Figure 3(d). The equilibrium points in (a) are not related to the equilibrium points of 1 . 